\title{
The prevalence of obstructive sleep apnoea and its association with aortic dilatation in Marfan's syndrome
}

\author{
M Kohler, ${ }^{1}$ E Blair, ${ }^{2}$ P Risby, ${ }^{2}$ A H Nickol, ${ }^{1}$ P Wordsworth, ${ }^{3}$ C Forfar, ${ }^{4}$ J R Stradling
}

${ }^{1}$ Oxford Centre for Respiratory Medicine, Churchill Hospital, Oxford, UK; ${ }^{2}$ Department of Clinical Genetics, Churchill Hospital, Oxford, UK; ${ }^{3}$ Department of Clinical Rheumatology, Nuffield Orthopaedic Hospital, Oxford, UK; ${ }^{4}$ Department of Cardiovascular Medicine, John Radcliffe Hospital, Oxford, UK

Correspondence to: Dr M Kohler, Oxford Centre for Respiratory Medicine, Oxford Radcliffe Hospitals, Churchill Hospital Campus, Headington, Oxford 0X3 7LJ, UK;

Malcolm.K@bluewin.ch

Received 5 June 2008 Accepted 3 September 2008 Published Online First 13 October 2008

\begin{abstract}
Background: Craniofacial abnormalities and increased pharyngeal collapsibility due to abnormal connective tissue suggest the possibility of an increased prevalence of obstructive sleep apnoea (OSA) in patients with Marfan's syndrome but the actual prevalence is uncertain. Aortic dilatation and dissection are life threatening manifestations of Marfan's syndrome and case reports have suggested a possible association with OSA but data from cohort studies are not available.
\end{abstract}

Methods: A sleep study was performed in 61 patients with Ghent criteria positive Marfan's syndrome (mean age 38.3 (SD 12.9) years; 37 females) and in 26 control subjects matched for age, gender, height and weight. OSA was defined using two conventional levels of apnoea-hypopnoea index (AHI), >5 and >15/h. In patients with Marfan's syndrome, aortic root diameter was measured by echocardiography.

Results: More patients with Marfan's syndrome than controls had OSA (AHI >5, 32.8\% compared with 11.5\%, mean difference $+21.3 \%$, $95 \% \mathrm{Cl} 4.2 \%$ to $38.3 \%$, $\mathrm{p}=0.04 ; \mathrm{AHI}>15,18.0 \%$ compared with $0 \%$, mean difference $+18.0 \%, 95 \% \mathrm{Cl} 8.4 \%$ to $27.7 \%, p=0.02$ ). $\mathrm{AHI}$ was correlated with aortic root diameter $(r=0.50,95 \%$ $\mathrm{Cl} 0.26$ to $0.69, p=0.0003$ ), and mean aortic root diameter was significantly greater in patients with OSA (4.5 (SD 0.6) cm) compared with those without OSA (3.7 (0.6) $\mathrm{cm}$ ) (mean difference $0.8 \mathrm{~cm}, 95 \% \mathrm{Cl} 0.4$ to $1.2 \mathrm{~cm}, \mathrm{p}<0.0001$ ).

Conclusions: In patients with Marfan's syndrome, the prevalence of OSA is considerably higher than in matched control subjects. OSA may be a risk factor for aortic root dilatation in Marfan's syndrome.

Marfan's syndrome is an autosomal dominant inherited multisystem disorder. It is caused by mutations in the FBN1 gene localised on chromosome $15 \mathrm{q} 21$, which encodes the matrix protein fibrillin 1. The incidence of Marfan's syndrome is estimated to be $2-3$ per 10000 individuals. ${ }^{1}$ To date there is no rapid and efficient molecular diagnostic test to identify individuals with the disease and thus the diagnosis primarily depends on a combination of major and minor clinical findings defined in the "Ghent criteria". ${ }^{3}$ The hallmark clinical features are noted in the cardiovascular, ocular and skeletal systems.

Aortic root dilatation and subsequent dissection are the commonest life threatening manifestations of Marfan's syndrome. Dilatation of the aortic root can begin in childhood or early adulthood and increases at an unpredictable rate. ${ }^{45}$ It is still a matter of debate which factors contribute to rapid progression of aortic root dilatation.

The high prevalence of craniofacial dysmorphisms and an increased upper airway collapsibility ${ }^{6}$ could predispose to an increased prevalence of obstructive sleep apnoea (OSA) in patients with Marfan's syndrome but there is only very limited information in the literature concerning the actual prevalence of OSA in this patient group. Cistulli and Sullivan ${ }^{7}$ reported a prevalence of $64 \%$ (defined by a apnoea-hypopnoea index (AHI) >5) but interpretation of this finding is limited as only 25 subjects with Marfan's syndrome were included in the study.

A possible causal link between OSA and aortic dilatation in patients with Marfan's syndrome has been suggested in two case reports. ${ }^{89}$ Possible underlying pathophysiological mechanisms are post-apnoea reflex sympathetic activation and consequent marked increases in blood pressure, ${ }^{10}$ which produce shear stresses in blood vessel walls-forces that are thought to cause vascular wall damage. ${ }^{11}$ Furthermore, largely negative intrathoracic pressure swings, which are produced during obstructive apnoeas, increase transaortic pressures $^{12}$ and may therefore accelerate aortic dilatation. To date, there are no data from cohort studies that have investigated the possible association of OSA with aortic root dilatation in patients with Marfan's syndrome.

We have addressed this issue by performing a case control study to evaluate the prevalence of OSA and its possible correlation with aortic root dilatation in a large cohort of patients with Marfan's syndrome.

\section{METHODS}

\section{Patients and controls}

Patients fulfilling the Ghent criteria ${ }^{2}{ }^{3}$ for Marfan's syndrome and attending their yearly clinical assessment at our institution were asked to participate in the study. Control subjects were recruited from the local population by an advertisement which was placed in the Oxford Radcliffe Hospitals NHS Trust sites and selected from among the volunteers to match subjects with Marfan's syndrome as closely as possible for average/frequency of age, gender, height and weight in order to compare the prevalence of OSA. Subjects were eligible if they were between 18 and 75 years of age, not pregnant and not treated with continuous positive airway pressure (CPAP). The study was approved by the Oxford research ethics committee (REC No 07/01607/6) 


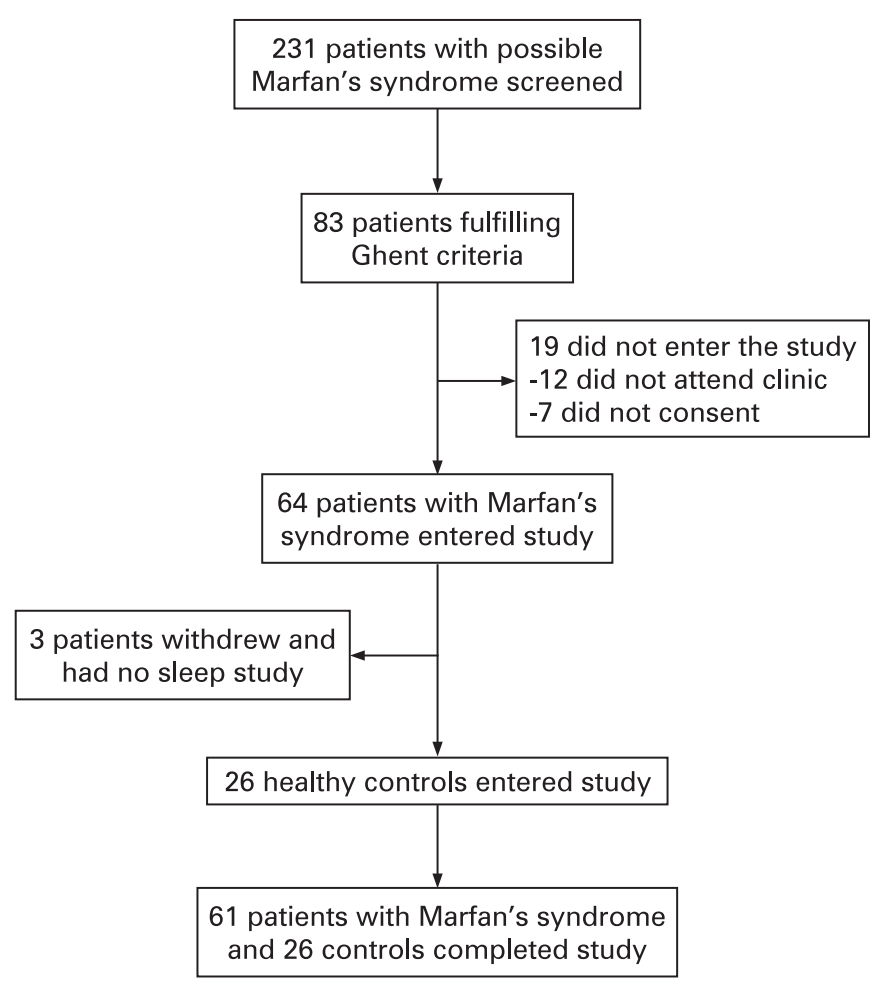

Figure 1 Study profile.

and written informed consent was obtained from all participants.

\section{Measurements}

\section{Anthropometrics and blood pressure}

The following anthropometric parameters were measured in all participants: height, weight, neck circumference, cricomental distance, palatal height, and maxillary and mandibular intermolar distance by callipers according to the method described by Kushida and colleagues. ${ }^{13}$ Tonsillar enlargement was assessed as previously described (class I, tonsils absent; class II, tonsils do not extend beyond the palatopharyngeal arch; class III, tonsils at the palatopharyngeal arch; class IV, tonsils extend beyond the palatopharyngeal arch). ${ }^{14}$ Palatal position or tongue size was assessed by the Mallampati score (class I, all of the oropharynx including tonsils, pillars, soft palate and tip of the uvula are visible; class II, tonsils' upper pole and uvula visible; class III, part of the uvula and soft palate visible; class IV, hard palate and part of soft palate are barely visible). ${ }^{15} 16$ The facial profile was categorised as retrognathic, neutral or prognathic in Frankfurt position, ${ }^{17}$ and the dental overbite was measured by a calliper, as previously described. ${ }^{14}$

Blood pressure and heart rate were measured in the sitting position with a standard digital automatic monitor (Omron Healthcare Company, Kyoto, Japan). The mean value of three readings was used for analysis.

\section{Sleep studies and questionnaire}

Subjective sleepiness was assessed using the Epworth Sleepiness Scale (ESS) questionnaire. Home sleep studies were performed using the ApneaLink device (ResMed, MAP Medicine Technology, Martinsried, Germany). The device records the patient's nasal respiratory pressure signal and finger oximetry during sleep, and has been validated as an accurate instrument
Table 1 Participant's characteristics

\begin{tabular}{lcc}
\hline & $\begin{array}{l}\text { Marfan's } \\
(\mathbf{n = 6 1 )}\end{array}$ & $\begin{array}{l}\text { Controls } \\
(\mathbf{n = 2 6 )}\end{array}$ \\
\hline Age (years) & $38.3(12.9)$ & $37.2(9.8)$ \\
Sex (W/M) & $37 / 24$ & $14 / 12$ \\
Height $(\mathrm{m})$ & $1.80(0.11)$ & $1.80(0.10)$ \\
Weight $(\mathrm{kg})$ & $81.4(20.5)$ & $78.1(15.0)$ \\
BMI $\left(\mathrm{kg} / \mathrm{m}^{2}\right)$ & $25.1(5.9)$ & $24.0(4.3)$ \\
Systolic BP $(\mathrm{mm} \mathrm{Hg})$ & $123.7(14.2)$ & $118.8(11.9)$ \\
Diastolic BP $(\mathrm{mm} \mathrm{Hg})$ & $76.2(11.0)$ & $74.4(9.8)$ \\
Heart rate $(/ \mathrm{min})$ & $62.5(9.9)$ & $65.0(9.8)$ \\
\hline
\end{tabular}

Values are mean (SD).

$\mathrm{BMI}$, body mass index; BP, blood pressure.

to detect snoring, apnoea-hypopnoea and oxygen desaturations. ${ }^{18}$ The results of the sleep study were scored automatically with dedicated software (ResMed, MAP Medicine Technology), with manual review to ensure accuracy of the data. Apnoeas were defined as a cessation of airflow lasting $>10 \mathrm{~s}$ and hypopnoeas as a reduction in airflow of at least $50 \%$ lasting $>10 \mathrm{~s}$, associated with a drop in oxygen saturation of $>4 \%$. Snoring severity was quantified as the number of snoring events per hour of study. OSA severity was quantified as the number of apnoeas-hypopnoeas (AHI) and oxygen desaturations $>4 \%$ per hour of study (ODI).

\section{Aortic root diameter measurements}

In all patients with Marfan's syndrome, echocardiography was performed by the same cardiac ultrasound technician who was not involved in the analysis of the study. Aortic root diameter was measured in the parasternal long axis view at end diastole (peak of $\mathrm{R}$ wave on electrocardiogram) and at end systole ( $\mathrm{T}$ wave on electrocardiogram) by two dimensional, $M$ mode echocardiography with a commercially available cardiac ultrasound system (Sonos 4500; Philips Healthcare, UK) using a 3.5 $\mathrm{MHz}$ transducer. The diameter of the aorta was assessed at several levels: at the left ventricular outflow tract, sinuses of Valsalva, supraaortic ridge and at the proximal ascending aorta, 1-2 cm above the supraaortic ridge, as described previously. ${ }^{19}$ The maximal diameter of the aortic root from these four assessments and the maximal diameter corrected for age and body surface area (predicted maximal diameter in per cent) were used for further statistical analysis. ${ }^{19}$

\section{Data analysis}

Data are expressed as means (SD). All statistical analyses were performed with Statistica V6.0 (StatSoft, Tulsa, Oklahoma, USA). Non-normally distributed data were normalised by square root transformation for statistical analysis. Demographics, results of the sleep study and echocardiography were compared by independent $t$ tests. In order to define the prevalence of OSA, two conventional threshold levels of AHI, $>5$ and $>15 / h$, were used. The threshold of $>5$ was also used for subgroup comparisons of snoring events, subjective daytime sleepiness and aortic root diameter in patients with and without OSA. For comparison of frequencies, the $\chi^{2}$ test of independence was used. Pearson's correlation analysis and multiple linear regression analysis were used to evaluate the relationships between the apnoea index (AI), AHI, ODI, anthropometrics and maximal aortic root diameter. A p value $<0.05$ was considered to be statistically significant. 
Table 2 Head and neck anthropometrics

\begin{tabular}{lccc}
\hline & $\begin{array}{c}\text { Marfan's } \\
(\mathbf{n}=\mathbf{6 1})\end{array}$ & $\begin{array}{c}\text { Controls } \\
(\mathbf{n}=\mathbf{2 6})\end{array}$ & p Value \\
\hline Neck circumference $(\mathrm{cm})$ & $36.5(3.6)$ & $36.6(4.2)$ & 0.85 \\
Cricomental distance $(\mathrm{cm})$ & $7.7(1.2)$ & $8.1(0.9)$ & 0.078 \\
Dental overbite $(\mathrm{mm})$ & $3.5(2.7)$ & $3.9(1.7)$ & 0.48 \\
Palatal height $(\mathrm{cm})$ & $4.0(0.7)$ & $3.7(0.7)$ & 0.029 \\
Maxillar intermolar distance $(\mathrm{cm})$ & $3.1(0.4)$ & $3.5(0.5)$ & $<0.0001$ \\
Mandibular intermolar distance $(\mathrm{cm})$ & $2.9(0.4)$ & $3.6(0.5)$ & $<0.0001$ \\
\% Retrognathic & 21.3 & 15.4 & 0.26 \\
Mallampati classes (\%)* & $30 / 44 / 18 / 8$ & $38 / 50 / 12 / 0$ & 0.36 \\
Tonsillar size classes (\%)* & $8 / 74 / 11 / 7$ & $0 / 77 / 19 / 4$ & 0.36 \\
\hline
\end{tabular}

Values are mean (SD).

*Percentage of subjects in each of the four classes (I/II/III/IV).

\section{RESULTS}

\section{Study profile and participants characteristics}

Figure 1 shows the study profile. Sixty-one patients with Ghent criteria positive Marfan's syndrome and 26 controls completed the study protocol. Patients with Marfan's syndrome were recruited from 41 unrelated families (no apparent relationships in their pedigree) of which 32 families provided one patient and nine families provided two or multiple patients (mostly first degree relatives). The two groups were similar (no statistically significant difference) regarding age, gender distribution, height, weight, body mass index (BMI), blood pressure and heart rate (table 1).

Patients with Marfan's syndrome were not different from control subjects regarding neck circumference, cricomental distance, dental overbite, Mallampati and tonsillar size score, and retrognathic face profile but palatal height was significantly greater and maxillar/mandibular intermolar distance was significantly smaller in patients with Marfan's syndrome (table 2).

\section{Sleep study, subjective sleepiness and predictors of OSA}

Sleep study and subjective sleepiness

AHI and ODI were significantly higher in patients with Marfan's syndrome compared with control subjects (table 3). Twenty patients with Marfan's syndrome (nine females) had an AHI $>5$ compared with three subjects in the control group (one female). Eleven patients with Marfan's syndrome had an AHI $>15$ compared with none in the control group. Eighteen patients with Marfan's syndrome had an ODI $>5$ compared with only one patient in the control group (table 3, fig 2). Inspection of the data showed that the 20 cases with an AHI $>5$ were distributed across 16 of the 41 families.

As expected, Marfan's patients with an AHI $>5$ had more snoring events per hour (83.0 (94.8)/h) than patients with an
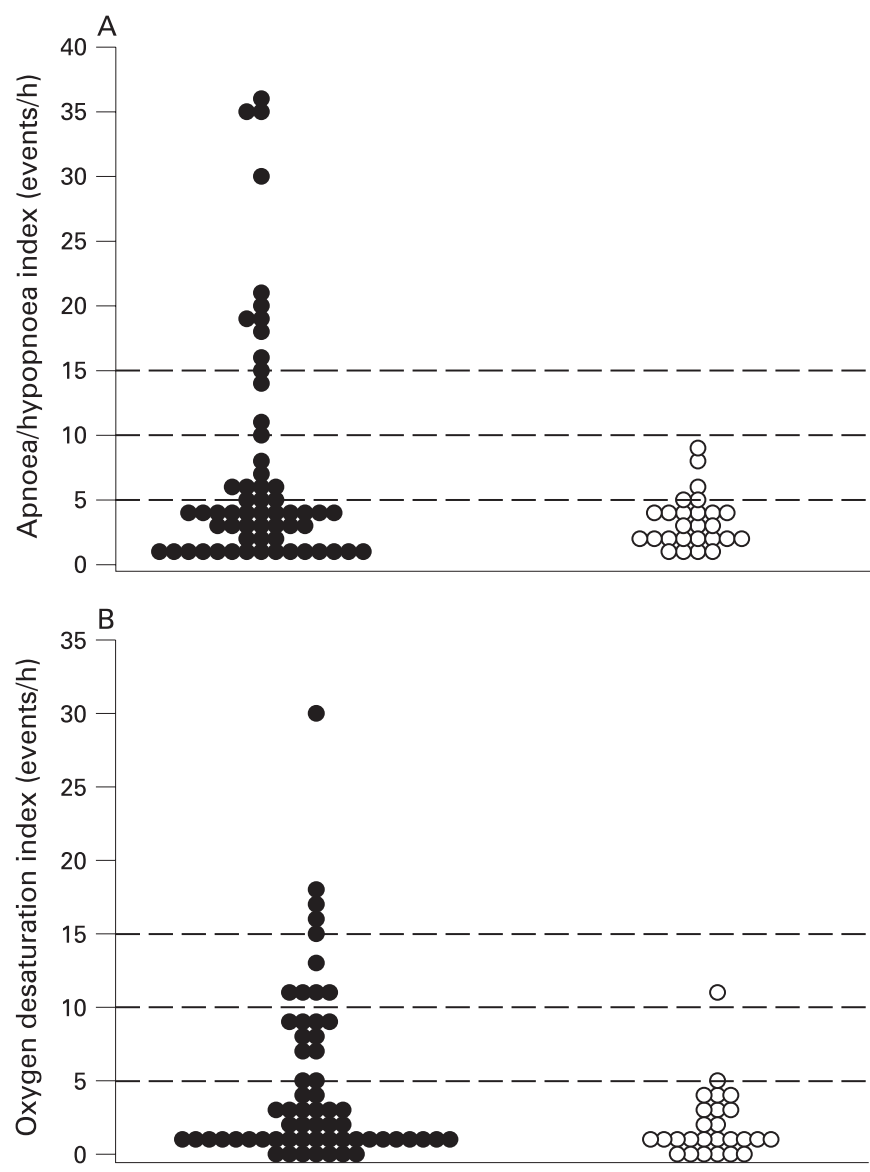

Figure 2 Apnoea-hypopnoea index (A) and oxygen desaturation index (ODI) (B) in patients with Marfan's syndrome (filled symbols) and controls (open symbols). Significantly more patients with Marfan's syndrome compared with the control group had an apnoea-hypopnoea index of $>5(32.8 \%$ vs $11.3 \%, p=0.04)$ and $>15(18.0 \%$ vs $0 \%$, $\mathrm{p}=0.02)$, and an ODI $>5(29.5 \%$ vs $3.9 \%, \mathrm{p}=0.008)$.

AHI $<5(28.9(49.1) / h)$ indicating that apnoeas were of the obstructive type (mean difference 54.1 events, 95\% CI 17.4 to 90.8 events, $\mathrm{p}=0.006$ ).

Subjective daytime sleepiness assessed by the ESS was higher in patients with Marfan's syndrome compared with controls (table 3).

Patients with an AHI $>5$ did not have a higher ESS (7.6 (4.3)) than patients with an AHI $<5(7.9(5.5))$ (mean difference -0.3 , $95 \% \mathrm{CI}-3.1$ to $+2.5, \mathrm{p}=0.83)$.

Table 3 Sleep study and subjective sleepiness

\begin{tabular}{|c|c|c|c|c|c|}
\hline & $\begin{array}{l}\text { Marfan's } \\
(\mathrm{n}=61)\end{array}$ & $\begin{array}{l}\text { Controls } \\
(n=26)\end{array}$ & Difference & $\begin{array}{l}95 \% \mathrm{Cl} \text { of } \\
\text { difference }\end{array}$ & p Value \\
\hline $\mathrm{AHI}$ & $5.5(0.9-13.9)$ & $3.0(1.5-5.2)$ & +0.37 & 0.003 to 1.37 & 0.03 \\
\hline$\%$ patients with $\mathrm{AHI}>5$ & 32.8 & 11.5 & +21.3 & 4.2 to 38.3 & 0.04 \\
\hline$\%$ patients with $\mathrm{AHI}>15$ & 18.0 & 0 & +18.0 & 8.4 to 27.7 & 0.02 \\
\hline ODI & $3.2(0.3-9.1)$ & $1.3(0.1-3.8)$ & +0.41 & 0.01 to 1.37 & 0.02 \\
\hline$\%$ patients with ODI $>5$ & 29.5 & 3.9 & +25.7 & 12.0 to 39.3 & 0.008 \\
\hline ESS & $7.8(5.1)$ & $4.6(3.2)$ & +3.2 & 1.1 to 5.4 & 0.004 \\
\hline
\end{tabular}

Values for $\mathrm{AHI}$ and $\mathrm{ODI}$ are mean and $1 \mathrm{SD}$ range (via square root transformation, and back-transformation, to normalise the distribution). Values for ESS are mean (SD).

Because of displacement of the nasal cannulae, AHI could not be calculated in two patients with Marfan's syndrome; the ODI was $<5$ in both subjects.

AHI, apnoea-hypopnoea index; ESS, Epworth Sleepiness Scale; ODI, oxygen desaturation index. 


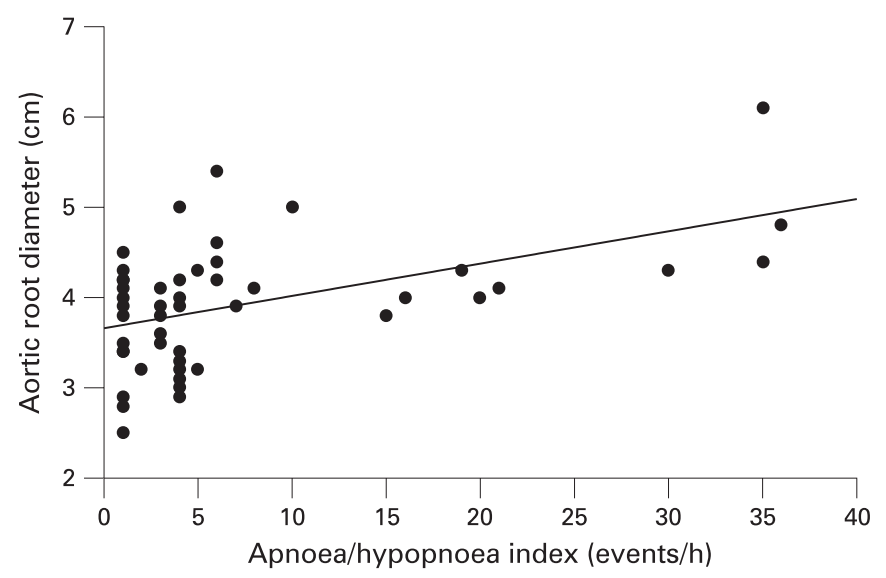

Figure 3 In patients with Marfan's syndrome, the apnoea-hypopnoea index showed a strong relationship with the diameter of the aortic root $(r=0.50,95 \% \mathrm{Cl} 0.26$ to $0.69, p=0.0003)$. The solid line represents the regression line according to the function: aortic root diameter $=3.66+$ $0.36 \times$ apnoea/hypopnoea index.

\section{Predictors of OSA}

There was a significant correlation between AHI and age $(r=0.37, p=0.004)$, BMI $(r=0.54, p=0.00001)$ and neck circumference $(r=0.45, p=0.0004)$ in patients with Marfan's syndrome. In a multiple linear regression model, 33\% of the variation in AHI could be explained by these factors $\left(r^{2}=0.33\right.$, $\mathrm{p}<0.0001)$ although only BMI appeared to be an independent predictor of AHI.

ODI was significantly correlated with age $(r=0.44$, $p=0.0004)$, BMI (0.70, $p<0.0001)$ and neck circumference $(r=0.43, p=0.0007)$. In a multiple linear regression model, $54 \%$ of the variation in ODI could be explained by these factors $\left(\mathrm{r}^{2}=0.54, \mathrm{p}<0.0001\right)$, and again only BMI was an independent predictor of ODI.

\section{Aortic root diameter}

Of the 61 patients with Marfan's syndrome, 12 patients $(19.7 \%)$ had previously undergone surgery for aortic root dilatation. In non-operated patients, mean aortic root diameter was significantly higher in subjects with an AHI $>5(\mathrm{n}=16,4.5(0.6) \mathrm{cm})$ than in subjects with an AHI $<5(\mathrm{n}=33,3.7(0.6) \mathrm{cm})$ (mean difference $+0.8 \mathrm{~cm}, 95 \%$ CI 0.4 to $1.2 \mathrm{~cm}, \mathrm{p}<0.0001)$. Per cent predicted aortic root diameter (corrected for age and body surface area) was higher in patients with AHI >5 (127.8 $(19.3) \%)$ than in patients with AHI <5 (116.8 (15.9)\%) (mean difference $11 \%$, 95\% CI 0.6 to $21.5 \%, p=0.039)$.

In non-operated patients, aortic root diameter was strongly correlated with AI and AHI $(r=0.50,95 \%$ CI 0.26 to 0.69 , $\mathrm{p}=0.0003$ for both correlations) (fig 3) and, as expected, with body surface area $(r=0.47,95 \%$ CI 0.22 to $0.66, p=0.0007)$ and age $(r=0.40,95 \%$ CI 0.13 to $0.61, p=0.005)$. When aortic root diameter was expressed as per cent predicted, it was still significantly correlated with AI ( $r=0.32,95 \%$ CI 0.04 to 0.55 , $p=0.028)$ whereas the correlation with AHI $(r=0.27,95 \%$ CI -0.01 to $0.51, p=0.068)$ did not quite attain statistical significance.

\section{DISCUSSION}

This is the first large case control study on the prevalence of OSA in patients with Marfan's syndrome showing a considerably higher frequency of OSA in patients with Marfan's syndrome compared with matched control subjects using either a threshold for AHI of $>5$ or $>15$. This is also the first study providing data on the relationship between OSA and aortic root dilatation in patients with Marfan's syndrome. We found an association between the severity of OSA and the diameter of the aortic root, which suggests that OSA may be a risk factor for aortic root dilatation in patients with Marfan's syndrome.

In this study, we found that approximately $30 \%$ of all patients with Marfan's syndrome had an AHI and ODI of $>5$, which is a considerably higher prevalence of OSA than in our matched controls $(3.9 \%$ had an ODI $>5$ ) or in a UK population based epidemiological study $(5 \%$ had an ODI $>5) .{ }^{20}$ However, Cistulli and Sullivan ${ }^{7}$ reported that 16 of 25 patients (64\%) with Marfan's syndrome had OSA (defined as AHI >5). A possible explanation for the very high prevalence of OSA found in the latter study may be selection bias due to the relatively small number of randomly recruited patients whereas we screened all patients attending the Oxford Marfan's clinic for eligibility and invited every patient with Ghent positive Marfan's syndrome to take part in the study. Another contributory explanation may be the higher percentage of females in our study $(60 \%$ compared with $52 \%$ in the study by Cistulli and Sullivan $)^{7}$ as the prevalence of OSA is known to be lower in women than in men. ${ }^{21}$ In the only other study on the prevalence of sleep disorders in Marfan's syndrome, Verbraecken and colleagues ${ }^{22}$ found that four of 15 patients (27\%) had features and symptoms of sleep apnoea although this study was based on questionnaires only.

A high frequency of craniofacial abnormalities and an increased upper airway collapsibility, resulting from the abnormally lax connective tissue, have previously been suggested as possible underlying causes for the high prevalence of OSA found in patients with Marfan's syndrome. ${ }^{63}$ In contrast with an earlier uncontrolled study in 15 patients with Marfan's syndrome, ${ }^{23}$ we found that patients with Marfan's syndrome were not different from control subjects regarding the frequency of a retrognathic facial profile, mean neck circumference, cricomental distance or Mallampati score, all of which are clinical features well known to be associated with OSA. ${ }^{24} 25$ The shorter intermolar distance and the greater palatal height we found in patients with Marfan's syndrome in comparison with control subjects are unlikely to contribute to the pathogenesis of OSA as there was no correlation between these measures and OSA severity, assessed by AHI, in our cohort (data not shown). Therefore, it seems that the higher prevalence of OSA found in patients with Marfan's syndrome is more likely to be the result of an increased upper airway collapsibility rather than of craniofacial abnormalities.

The high prevalence of OSA in Marfan's syndrome raises the question of how should an individual be assessed for potential OSA. We identified BMI as the only independent predictor of OSA in patients with Marfan's syndrome and unexpectedly the degree of subjective sleepiness was not related to the severity of OSA. This suggests that simply assessing a patient's subjective sleepiness is not a useful method to screen for OSA in a cohort of Marfan's patients. Therefore, a detailed medical history, including questions on snoring, apnoeas and sleepiness, together with an assessment of BMI, is warranted in every patient with Marfan's syndrome and may help the clinician to decide whether a sleep study should be performed.

Aortic root dilatation and subsequent dissection are the most life threatening manifestations of Marfan's syndrome. Dilatation of the aortic root can begin in childhood or early adulthood and increases at an unpredictable rate. ${ }^{45}$ It is still a matter of debate which factors contribute to rapid progression 
of aortic root dilatation. This is the first study in which the potential relationship between OSA and aortic root dilatation in patients with Marfan's syndrome has been investigated. Our data show that the severity of OSA is associated with an increased aortic root diameter (fig 3), and patients with OSA had a larger aortic root than patients without OSA. These results are corroborated by the findings of two case reports in which treatment of OSA with CPAP was associated with attenuation of aortic root dilatation in three patients with Marfan's syndrome. ${ }^{89}$ This suggests that OSA promotes aortic dilatation in patients with Marfan's syndrome. To date, the underlying mechanisms through which OSA may promote aortic dilatation in these patients are not clear. OSA has been shown to be associated with increased diurnal blood pressure as well as with recurrent surges in blood pressure during apnoeic events, ${ }^{26}$ which is the main risk factor for aortic dilatation and dissection. ${ }^{27}$ In addition, obstructive apnoeas are associated with repeated inspiratory effort against the collapsed upper airway, causing recurrent large subatmospheric intrathoracic pressure swings (sometimes over $60 \mathrm{~mm} \mathrm{Hg}$ ) and thereby producing extensive shear stresses on intrathoracic structures, including the ascending aorta. ${ }^{28}$ This hypothesis is supported by the findings of Peters et al who reported increased aortic diameters during obstructive apnoeas in an animal model. ${ }^{29} 30$ Furthermore, Sampol and colleagues ${ }^{31}$ recently found that (non-Marfan's) patients with dissection of the thoracic aorta had a higher apnoea-hypopnoea index versus well matched hypertensive control subjects without dissection (28 (30) vs 11 $(10) ; p=0.03)$. However, a randomised controlled trial on the effects of CPAP on aortic root diameter in patients with OSA and Marfan's syndrome is required to prove if treatment of OSA indeed attenuates the progression of aortic dilatation.

In conclusion, we have shown that OSA is highly prevalent in patients with Marfan's syndrome. It is not possible to reliably predict the presence of OSA in these patients by assessment of subjective sleepiness or facial morphological features. OSA may be a risk factor for aortic dilatation in patients with Marfan's syndrome, and randomised controlled trials investigating the effects of continuous positive airway pressure on aortic dilatation are warranted to definitely prove this relationship.

Funding: MK is a recipient of a European Respiratory Society (No 118) and University of Zurich, Switzerland, research fellowship.

Competing interests: None.

Ethics approval: The study was approved by the Oxford research ethics committee (REC No 07/01607/6).

\section{REFERENCES}

1. Judge DP, Dietz HC. Marfan's syndrome. Lancet 2005;366:1965-76.

2. De Paepe A, Devereux RB, Dietz HC, et al. Revised diagnostic criteria for the Marfan syndrome. Am J Med Genet 1996:62:417-26.
3. Loeys B, De Backer J, Van Acker P, et al. Comprehensive molecular screening of the FBN1 gene favors locus homogeneity of classical Marfan syndrome. Hum Mutat 2004;:24:140-6

4. Hwa J, Richards JG, Huang $\mathrm{H}$, et al. The natural history of aortic dilatation in Marfan syndrome. Med J Aust 1993;158:558-62.

5. Jeremy RW, Huang $\mathrm{H}$, Hwa J, et al. Relation between age, arterial distensibility, and aortic dilatation in the Marfan syndrome. Am J Cardiol 1994;74:369-73.

6. Cistulli PA, Sullivan CE. Sleep apnea in Marfan's syndrome. Increased upper airway collapsibility during sleep. Chest 1995;108:631-5.

7. Cistulli PA, Sullivan CE. Sleep-disordered breathing in Marfan's syndrome. Am Rev Respir Dis 1993;147:645-8.

8. Cistulli PA, Wilcox I, Jeremy R, et al. Aortic root dilatation in Marfan's syndrome. A contribution from obstructive sleep apnea? Chest 1997;111:1763-6.

9. Verbraecken J, Paelinck BP, Willemen M, et al. Aortic root diameter and nasal intermittent positive airway pressure treatment in Marfan's syndrome. Clin Genet 2003;63:131-4.

10. Zwilich C, Sinoway L. Surges of muscle sympathetic nerve activity during obstructive apnea are linked to hypoxemia. J Appl Physiol 1995;79:581-8.

11. Lovett JK, Rothwell PM. Site of carotid plaque ulceration in relation to direction of blood flow: an angiographic and pathological study. Cerebrovasc Dis 2003; 16:369-75.

12. Issa FG, Sullivan CE. Upper airway closing pressures in snorers. J Appl Physiol 1984;57:528-35

13. Kushida CA, Efron B, Guilleminault C. A predictive morphometric model for the obstructive sleep apnea syndrome. Ann Intern Med 1997:127:581-7.

14. Tsai WH, Remmers JE, Brant R, et al. A decision rule for diagnostic testing in obstructive sleep apnea. Am J Respir Crit Care Med 2003;167:1427-32.

15. Mallampati SR, Gatt SP, Gugino LD, et al. A clinical sign to predict difficult tracheal intubation. Can Anaesth Soc J 1985;32:429-34.

16. Samsoon GLT, Young JRB. Difficult tracheal intubation: a retrospective study. Anaesthesia 1987;42:487-90.

17. Ridley MB. Aesthetic facial proportions. In: Papel ID, Nachlis NE, eds. Facial plastic and reconstructive surgery. St Louis: Mosby-Year Book, 1992:106.

18. Wang $\mathbf{Y}$, Teschler $T$, Weinreich $G$, et al. Validation of microMESAM as screening device for sleep disordered breathing. Pneumologie 2003:57:734-40.

19. Roman MJ, Devereux RB, Kramer-Fox R, et al. Two-dimensional echocardiographic aortic root dimensions in normal children and adults. Am J Cardiol 1989;64:507-12.

20. Stradling JR, Crosby JH. Predictors and prevalence of obstructive sleep apnoea and snoring in 1001 middle aged men. Thorax 1991;46:85-9.

21. Young T, Peppard PE, Gottlieb DJ. Epidemiology of obstructive sleep apnea: population health perspective. Am J Respir Crit Care Med 2002;165:1217-39.

22. Verbraecken J, Declerck A, Van de Heyning $P$, et al. Evaluation for sleep apnea in patients with Ehlers-Danlos syndrome and Marfan: a questionnaire study. Clin Genet 2001;60:360-5

23. Cistulli PA, Gotsopoulos H, Sullivan CE. Relationship between craniofacial abnormalities and sleep-disordered breathing in Marfan's syndrome. Chest 2001; 120:1455-60.

24. Jamieson A, Guilleminault C, Partinen M, et al. Obstructive sleep apneic patients have craniomandibular abnormalities. Sleep 1986;9:469-77.

25. Davies RJ, Ali NJ, Stradling JR. Neck circumference and other clinical features in the diagnosis of the obstructive sleep apnoea syndrome. Thorax 1992;47:101-5.

26. Somers VK, Kyken ME, Clary MP, et al. Sympathetic neural mechanisms in obstructive sleep apnea. J Clin Invest 1995;96:1897-904.

27. Hagan PG, Nienaber CA, Isselbacher EM, et al. The international registry of acute aortic dissection (IRAD): new insights into an old disease. JAMA 2000;283:897-903.

28. Kimoff RJ, Cheong TH, Olha AE, et al. Mechanisms of apnea termination in obstructive sleep apnea: role of chemoreceptor and mechanoreceptor stimuli. Am J Respir Crit Care Med 1994;149:707-14.

29. Peters J, Kindred MK, Robotham JL. Transient analysis of cardiopulmonary interactions: I: diastolic events. J Appl Physiol 1988;64:1506-17.

30. Peters J, Kindred MK, Robotham JL. Transient analysis of cardiopulmonary interactions: II: systolic events. J Appl Physiol 1988;64:1518-26.

31. Sampol G, Romero 0, Salas A, et al. Obstructive sleep apnea and thoracic aorta dissection. Am J Respir Crit Care Med 2003;168:1528-31. 